\title{
Desfechos cirúrgicos e complicações de laparoscopias ginecológicas em hospital universitário brasileiro no período de 2014 a 2016
}

\section{Surgical outcomes and complications of gynecological laparoscopies in a Brazilian university hospital from 2014 to 2016}

Thaís Costa Gomes ${ }^{1}$. Kathiane Lustosa Augusto ${ }^{2}$. Silvana Lícia Nogueira Machado ${ }^{3}$. Mariana Cidade Amâncio $^{3}$. Gizelle Maria Moisés Monteiro³. Luana Ibiapina Machado³.

1 Residente de Ginecologia e Obstetrícia, Universidade Federal do Ceará (UFC). Médica, Maternidade Escola Assis Chateaubriand (MEAC), Fortaleza, Ceará, Brasil. 2 Mestre em Ciências Médico-cirúrgicas, Universidade Federal do Ceará (UFC), Programa de Ginecologia e Obstetrícia, Maternidade Escola Assis Chateaubriand (MEAC), Fortaleza, Ceará, Brasil. 3 Discente da Faculdade de Medicina, Universidade Federal do Ceará (UFC), Fortaleza, Ceará, Brasil.

\section{RESUMO}

Objetivo: coletar informações do prontuário de todas as pacientes que realizaram cirurgia laparoscópica ginecológica, afim de identificar quais cirurgias foram mais realizadas por videolaparoscopia e de avaliar as principais complicações cirúrgicas intra e pósoperatórias ocorridas. Metodologia: estudo retrospectivo realizado em hospital terciário universitário de Fortaleza, Ceará, Brasil, durante o período de setembro de 2014 a novembro de 2016. Resultados: os procedimentos ginecológicos laparoscópicos mais realizados durante o período estudado foram ooforoplastia, exérese de foco de endometriose, lise de aderências, salpingooforectomia, salpingectomia e laqueadura tubária. As complicações intraoperatórias ocorreram em 13 das 238 cirurgias realizadas no período referido, com 4 cirurgias convertidas para laparotomia. As complicações cirúrgicas observadas no pós-operatório imediato ocorreram em 89 casos no total, sendo 45 casos de dor, 13 casos de sangramento e 10 casos de intercorrências clínicas. Conclusão: existem complicações inerentes à prática cirúrgica e na cirurgia laparoscópica há complicações específicas desta técnica, estando algumas taxas de complicações do nosso estudo semelhantes e outras menores que as taxas relatadas na literatura mundial. É essencial comunicar nossas complicações para contribuir para um melhor conhecimento sobre elas, assim como classificá-las e registrá-las apropriadamente.

Palavras-chave: Ginecologia. Laparoscopia. Cirurgia.

\section{ABSTRACT}

Objective: To collect information from the charts of all patients who underwent laparoscopic gynecological surgery, in order to identify which surgeries were performed by videolaparoscopy and to evaluate the main intra and postoperative surgical complications. Methodology: Retrospective study performed at a university tertiary hospital in Fortaleza, Ceará, Brazil, from September 2014 to November 2016. Results: The most performed laparoscopic gynecological procedures during the period studied were oophoroplasty, excision of focus of endometriosis, lysis of adhesions, salpingooforectomy, salpingectomy, tubal ligation. Intraoperative complications occurred in 13 of the 238 surgeries performed in the referred period, with 4 surgeries converted to laparotomy. The surgical complications observed in the immediate postoperative period occurred in 89 cases in total, being 45 cases of pain, 13 cases of bleeding and 10 cases of clinical complications. Conclusion: There are complications inherent to the surgical practice and in laparoscopic surgery there are specific complications of this technique, being that some complication rates of our study are similar and other minor than the rates reported in the world literature. It is essential to communicate our complications to contribute to a better knowledge about them, as well as to classify and record them properly.

Keywords: Gynecology. Laparoscopy. Surgery.

Autor correspondente: Thaís Costa Gomes, Rua Paz, 455, Mucuripe, Fortaleza, Ceará. CEP: 60165-180.Telefone: +55 85987052310. E-mail: tatahcosta@yahoo.com.br

Conflito de interesses: Não há qualquer conflito de interesses por parte de qualquer um dos autores.

Recebido em: 28 Fev 2018; Revisado em: 08 Abr 2018; Aceito em: 15 Mai 2018. 


\section{INTRODUÇÃO}

A cirurgia laparoscópica remonta ao início dos anos 1900 e foi descrita pela primeira vez por Kelling em 1902. A laparoscopia ginecológica foi inicialmente utilizada como ferramenta para inspeção pélvica e diagnóstico. Atualmente, está sendo usada cada vez mais para grandes procedimentos operatórios. Tornou-se um pilar da gestão cirúrgica nos campos da ginecologia, cirurgia geral e urologia. ${ }^{1}$

Laparoscopia é um procedimento cirúrgico minimamente invasivo que nas últimas décadas apresentou aumento expressivo, evoluindo de um procedimento cirúrgico limitado, como cirurgias simples benignas, a uma ferramenta cirúrgica importante usada numa variedade de indicações. Hoje, a laparoscopia é um dos procedimentos cirúrgicos mais comuns realizados em muitas partes do mundo e técnicas laparoscópicas continuam a evoluir, principalmente como resultado de avanços tecnológicos. ${ }^{2}$

As vantagens principais da laparoscopia em relação à laparotomia são ocasionar menor trauma cirúrgico, menor sangramento intraoperatório, menor dor pós-operatória, recuperação pós-cirúrgica mais rápida, retorno mais cedo às atividades habituais e ao trabalho, menor tempo de hospitalização e menores cicatrizes. Além disso, ela reduz a taxa de infecções e a ocorrência de aderências pós-operatórias. ${ }^{3,4}$

A laparoscopia é um dos procedimentos cirúrgicos mais comuns realizados hoje nos Estados Unidos (EUA). Aproximadamente metade das 700.000 esterilizações tubárias bilaterais realizadas anualmente nos EUA são realizadas de forma laparoscópica. Além da laparoscopia diagnóstica, a endoscopia cirúrgica é utilizada para realizar procedimentos comuns, incluindo remoção de gravidezes ectópicas, tratamento de endometriose e lise de aderências pélvicas. Quase um terço das 600.000 histerectomias realizadas anualmente nos EUA são com o auxílio de um laparoscópio. Embora o papel ideal da laparoscopia na cirurgia ginecológica ainda não seja completamente definido, ela se tornou abordagem padrão para uma grande quantidade de procedimentos ginecológicos. ${ }^{2}$

Em sua essência, a laparoscopia permanece um procedimento intra-abdominal. Portanto, compartilha todos os riscos intraoperatórios e pós-operatórios da laparotomia, incluindo infecção e lesão de estruturas intra-abdominais adjacentes. Quando os principais procedimentos intra-abdominais são realizados laparoscopicamente (por exemplo, histerectomia), a dor e a morbidade pós-operatórias resultantes são ainda significativas. No entanto, como uma grande incisão abdominal é desnecessária, a dor e a morbidade pósoperatórias são sempre menos significativas do que a cirurgia maior semelhante realizada por laparotomia. ${ }^{2}$

Como em todos os aspectos da Medicina, a cirurgia laparoscópica requer experiência por parte do cirurgião, a fim de proporcionar às pacientes o melhor resultado possível. A anatomia visível através de um laparoscópio difere em perspectiva da apresentada nas cirurgias abertas.
Técnicas básicas, como a sutura, devem ser reaprendidas e há uma diminuição no sentido tátil, quando um instrumento é interposto entre a ponta do dedo e o tecido. A instrução adequada e a supervisão parecem prudentes à medida que um cirurgião sobe em sua curva de aprendizado. ${ }^{5}$

É importante enfatizar que o melhor resultado cirúrgico é obtido quando há precisa indicação da via cirúrgica previamente ao procedimento. ${ }^{6}$

As complicações podem ser definidas como um evento não esperado e/ou não planejado, que modificaria o curso usual da laparoscopia ou do período pós-operatório, que requeira outro tipo de tratamento como laparotomia, longa observação pósoperatória ou outra cirurgia. ${ }^{7}$

As complicações costumam ser agrupadas em maiores e menores. São consideradas maiores as complicações cujo grau de injúria requer a realização de laparotomia ou levam à morte. Complicações menores são lesões ou problemas que podem ser resolvidos durante a videolaparoscopia e que permitem a finalização do procedimento no mesmo ato cirúrgico. ${ }^{7}$

As complicações da cirurgia laparoscópica podem ser divididas principalmente em três grupos: complicações derivadas do pneumoperitôneo, complicações intraoperatórias e complicações pós-operatórias. ${ }^{8}$

Os procedimentos laparoscópicos tem riscos únicos, exemplo disso são os riscos oriundos da confecção do pneumoperitôneo. Apesar de pouco frequentes, as lesões vasculares e as lesões intestinais, por exemplo, são bastante temidas quando ocorrem durante a laparoscopia. ${ }^{9}$

O objetivo desse estudo, realizado em um hospital terciário universitário do Ceará, Brasil, é conhecer os desfechos cirúrgicos e as taxas de complicações das pacientes submetidas à laparoscopia para tratamento de doenças ginecológicas, .

\section{MATERIAL E MÉTODO}

Trata-se de estudo retrospectivo realizado em hospital terciário universitário de Fortaleza, Ceará, Brasil, durante o período de setembro de 2014 a novembro de 2016. Os dados foram coletados de informações de prontuário de todas as pacientes que realizaram cirurgia laparoscópica ginecológica no período referido.

Os aspectos avaliados foram os desfechos cirúrgicos relacionados aos tipos de procedimento realizados e as taxas de complicações destes procedimentos nas pacientes submetidas à laparoscopia. Não foram estabelecidos limites de idade ou quaisquer outros critérios de exclusão para a pesquisa, uma vez que o objetivo é fornecer um levantamento das cirurgias laparoscópicas ginecológicas.

A análise estatística foi feita pelo SPSS 16 através de média e mediana e porcentagem simples. O Comitê de Ética da Maternidade Escola Assis Chateaubriand aprovou o projeto 
(parecer de número 2.350.976). A pesquisa foi realizada de acordo com os critérios estabelecidos pela Declaração de Helsinki com as suas modificações.

\section{RESULTADOS}

Foram avaliadas 238 pacientes que realizaram cirurgia no período de setembro de 2014 a novembro de 2016. Foram contabilizados os procedimentos realizados, que pode ter sido mais de um durante o mesmo ato cirúrgico.
Os procedimentos ginecológicos laparoscópicos mais realizados durante o período estudado foram ooforoplastia (59 casos), exérese de foco de endometriose (48 casos), lise de aderências (43 casos), salpingooforectomia (37 casos), salpingectomia (30 casos), laqueadura tubária (27 casos), exérese de lesão de reto e/ou sigmoide (18 casos), histerectomia (17 casos), ooforectomia (15 casos), ureterólise bilateral (14 casos), miomectomia (11 casos) e laparoscopia diagnóstica (8 casos), em um total de 238 cirurgias (Tabela 1).

Tabela 1. Apresentação da quantidade dos procedimentos cirúrgicos laparoscópicos mais realizados em hospital universitário brasileiro no período de 2014 a 2016.

\begin{tabular}{|c|c|c|}
\hline Procedimentos & $n^{0}$ de casos & Porcentagem \\
\hline Ooforoplastia & 59 & $24,78 \%$ \\
\hline Exérese de foco de endometriose & 48 & $20,17 \%$ \\
\hline Lise de aderências & 43 & $18,06 \%$ \\
\hline Salpingooforectomia & 37 & $15,54 \%$ \\
\hline Salpingectomia & 30 & $12,60 \%$ \\
\hline Laqueadura tubária & 27 & $11,34 \%$ \\
\hline Exérese de lesão de reto e/ou sigmoide & 18 & $7,56 \%$ \\
\hline Histerectomia & 17 & $7,14 \%$ \\
\hline Ooforectomia & 15 & $6,30 \%$ \\
\hline Ureterólise bilateral & 14 & $5,88 \%$ \\
\hline Miomectomia & 11 & $4,62 \%$ \\
\hline Laparoscopia diagnóstica & 8 & $3,36 \%$ \\
\hline Drenagem de cisto ovariano & 4 & $1,68 \%$ \\
\hline Promontofixação & 3 & $1,26 \%$ \\
\hline Linfadenectomia pélvica bilateral & 3 & $1,26 \%$ \\
\hline Linfadenectomia retroperitoneal & 2 & $0,84 \%$ \\
\hline Recanalização tubária & 2 & $0,84 \%$ \\
\hline Exérese de lesão de peritôneo & 2 & $0,84 \%$ \\
\hline Apendicectomia & 2 & $0,84 \%$ \\
\hline Gonadectomia & 2 & $0,84 \%$ \\
\hline Correção de fístula vesico-vaginal & 2 & $0,84 \%$ \\
\hline Biópsias & 2 & $0,84 \%$ \\
\hline Exérese de cisto interligamentar & 1 & $0,42 \%$ \\
\hline Exérese de tumor retroperitoneal & 1 & $0,42 \%$ \\
\hline Exérese de cúpula vaginal/colo & 1 & $0,42 \%$ \\
\hline Exérese ligamentar & 1 & $0,42 \%$ \\
\hline Retirada de DIU da cavidade abdominal & 1 & $0,42 \%$ \\
\hline Drilling de ovário & 1 & $0,42 \%$ \\
\hline Salpingotomia & 1 & $0,42 \%$ \\
\hline Salpingooforoplastia & 1 & $0,42 \%$ \\
\hline Drenagem do cisto paratubário & 1 & $0,42 \%$ \\
\hline Drenagem do abscesso no fundo de saco posterior & 1 & $0,42 \%$ \\
\hline Sutura de bexiga & 1 & $0,42 \%$ \\
\hline Pesquisa de linfonodo sentinela & 1 & $0,42 \%$ \\
\hline Total & 363 & \\
\hline
\end{tabular}


As complicações intraoperatórias ocorreram em 13 casos (5,46\% do total), sendo 1 caso de acidente de punção, 1 caso de lesão de órgão, 1 caso de lesão de vaso, 3 pacientes necessitaram de transfusão sanguínea, 3 pacientes necessitaram de colocação de cateter duplo J e 4 cirurgias foram convertidas para laparotomia (Tabela 2).

As complicações cirúrgicas observadas no pós-operatório imediato ocorreram em 89 casos $(37,4 \%$ do total), sendo 45 casos de dor (33 casos de dor leve a moderada e 12 casos de dor intensa), 13 casos de sangramento, 10 casos de intercorrências clínicas, 6 casos de alteração intestinal, 4 casos de infecção (1 caso de infecção de ferida operatória, 1 caso de infecção urinária, 1 caso de infecção intestinal e 1 caso de sepse abdominal), 1 caso de deiscência e 1 caso de enfisema subcutâneo. Nove pacientes precisaram de acompanhamento em Unidade de Terapia Intensiva (UTI). Nenhuma paciente sofreu algum tipo de queimadura (Tabela 3 ).

Tabela 2. Complicações intraoperatórias em hospital universitário brasileiro no período de 2014 a 2016.

\begin{tabular}{lll}
\hline Complicações intraoperatórias & $\mathbf{n}^{\mathbf{0}}$ casos & Porcentagem \\
\hline Conversão para laparotomia & 4 & $30,77 \%$ \\
Transfusão sanguínea & 3 & $23,07 \%$ \\
Cateter duplo J & 3 & $23,07 \%$ \\
Acidente de punção & 1 & $7,69 \%$ \\
Lesão de vaso & 1 & $7,69 \%$ \\
Lesão de órgão & 1 & $7,69 \%$ \\
Total & 13 & \\
\hline
\end{tabular}

Tabela 3. Complicações cirúrgicas no pós-operatório imediato em hospital universitário brasileiro no período de 2014 a 2016.

\begin{tabular}{lll}
\hline Complicações cirúrgicas no pós-operatório imediato & $\mathbf{n}^{\mathbf{0}}$ de casos & Porcentagem \\
\hline Dor leve a moderada & 33 & $37,08 \%$ \\
Sangramento & 13 & $14,60 \%$ \\
Dor intensa & 12 & $13,48 \%$ \\
Intercorrências clínicas & 10 & $11,23 \%$ \\
Acompanhamento em UTI & 9 & $10,11 \%$ \\
Alteração intestinal & 6 & $6,74 \%$ \\
Infecção & 4 & $4,49 \%$ \\
Deiscência & 1 & $1,12 \%$ \\
Enfisema subcutâneo & 1 & $1,12 \%$ \\
Total & 89 & \\
\hline
\end{tabular}

\section{DISCUSSÃO}

Atualmente, a cirurgia videolaparoscópica oferece uma série de benefícios em relação à cirurgia aberta e está sendo empregada como rotina em alguns serviços de Ginecologia, em função da sua eficácia e segurança, porque consegue reunir em um só procedimento a propedêutica e a terapêutica. ${ }^{7}$

As principais complicações intraoperatórias são muitas vezes relacionadas aos métodos utilizados para a colocação de trocateres (manobras sem a visualização direta do operador, por exemplo) e para a criação do pneumoperitônio necessários para a laparoscopia. Esses riscos incluem complicações vasculares, complicações no trato urinário, complicações intestinais e outras complicaçoes mais raras (decorrentes do posicionamento da paciente, incisionais, de acidose metabólica por absorção peritoneal de gás carbônico etc). Além disso, o aumento das pressões intra-abdominais associadas à laparoscopia aumentam os riscos relacionados à anestesia, tais como a aspiração e o aumento da dificuldade de ventilação da paciente. Embora o risco de perda de sangue seja relativamente baixo para a maioria dos procedimentos laparoscópicos, uma perda de sangue potencialmente maciça pode ocorrer e é complicada pelo fato de que o controle da perda de sangue pode ser atrasado pelo tempo necessário para realizar uma laparotomia de emergência. ${ }^{9}$ No presente estudo, ocorreram 13 complicações intraoperatórias, que, quando comparadas ao total de cirugias realizadas, teve uma taxa de $5,46 \%$. Na literatura, a taxa de complicações varia de $0,2 \%$ e $10,3 \%,{ }^{10}$ correspondendo aos achados do nosso estudo.

A incidência de lesão intestinal é relatada em $0 \%$ a $0,5 \%$ dos casos. A incidência estimada de lesão ureteral durante a 
histerectomia laparoscópica é de 2,6 a 35 vezes mais comum $(0,2 \%$ a $6,0 \%)$ do que na histerectomia abdominal. A lesão ureteral representou $4,3 \%$ a $7 \%$ das complicações totais da laparoscopia. O tipo mais comum de lesão urinária durante a laparoscopia é a perfuração da bexiga, com uma incidência de $0,02 \%$ a $8,3 \%$. As lesões envolvendo o vaso epigástrico inferior são o tipo mais comum de complicação vascular. As lesões vasculares retroperitoneais são complicações fatais cuja incidência é estimada entre $0,04 \%$ e $0,5 \%$, sendo a maioria relacionada à entrada e independente da complexidade da cirurgia. Nesta situação, a aorta, a veia cava e, mais comumente, a ilíaca comum são atingidas. ${ }^{10}$

Segundo Beleña et al., a perfuração intestinal tem incidência variando entre $0,1 \%$ e $0,3 \%$ dos casos. Um dos problemas relacionados a esta complicação é a dificuldade intraoperatória para diagnosticá-lo, sendo a maioria das lesões $(70 \%$ dos casos) diagnosticadas no pós-operatório. ${ }^{8}$

Em um outro estudo, foi realizada uma análise de 262 histerectomias laparoscópicas, havendo uma taxa de complicações major de $1,5 \%$ e de complicações minor de $11,5 \%$, não tendo ocorrido nenhum caso de lesão ureteral, vesical, perfuração intestinal ou morte. ${ }^{11}$ Numa série de 1120 histerectomias laparoscópicas, Karaman et al. descreveram uma taxa de complicações major de $1 \%{ }^{11}$

No nosso estudo, houve uma taxa $0,42 \%$ de acidente de punção, $0,42 \%$ de lesão de órgão, $0,42 \%$ de lesão de vaso, $1,26 \%$ de transfusão sanguínea e $1,68 \%$ de conversão para laparotomia. Essas taxas mostram que as complicações relatadas em nosso estudo são menores que as taxas relatadas na literatura.

Leng $\mathrm{J}$ et al. publicaram um artigo que investigou retrospectivamente as complicações e os fatores associados em 1769 cirurgias laparoscópicas. Foram detectadas 17 complicações durante o pós-operatório, sendo 2 hemorragias intraperitoneais (11,7\% dos casos), 2 lesões intestinais $(11,7 \%$ dos casos), 4 paresias nervosas (23,5\% dos casos) e 9 patologias febris $(53 \%$ dos casos $){ }^{12}$

Um estudo exploratório e descritivo realizado no Central University Hospital of Dakar estudou uma série contínua de 128 laparoscopias ginecológicas realizadas entre os anos de 2006 e 2009. Concluiu-se que a taxa de conversão para laparotomia foi necessária em $7 \%$ dos casos, devido à extensão das adesões (3 casos), ao tratamento de alguma patologia associada ( 4 casos) ou à dificuldade técnica $(2$ casos $) .{ }^{13}$

Segundo Sokol et al., os seguintes fatores são associados à conversão da laparoscopia à laparotomia não intencional: inexperiência do cirurgião, nível de complexidade laparoscópica, índice de massa corporal superior a $30 \mathrm{~kg} / \mathrm{m}^{2}$, suspeita de malignidade, história de laparotomia, presença de adesões e dificuldade técnica intraoperatória. ${ }^{14}$

No nosso estudo, houve uma taxa de $1,68 \%$ de conversão para laparotomia, sendo menor que a taxa da literatura citada anteriormente. Dos 4 casos convertidos, 2 foram causados por insucesso da confecção do pneumoperitônio, 1 caso por suspeita de lesão intestinal e 1 caso devido à bloqueio firme em fossa ilíaca esquerda com alça de delgado à $20 \mathrm{~cm}$ da válvula ileocecal firmemente aderida ao útero.

A maioria das complicações ocorre no início do procedimento laparoscópico e estão associadas a erros técnicos ou falta de atenção durante a inserção da agulha e/ou trocartes na cavidade peritoneal. As lesões mais significantes são as intestinais (principalmente quando passam despercebidas no intraoperatório) e as vasculares, por trazerem consequências graves à paciente. ${ }^{9}$

Acredita-se que o dióxido de carbono sob o diafragma é o responsável por grande parte da dor experimentada após a laparoscopia. ${ }^{15} \mathrm{~A}$ dor no abdome superior, a dor do ombro e as dores posturais nas costas podem ser causadas devido ao gás retido na cavidade peritoneal. $\mathrm{O}$ dióxido de carbono é usado para expandir o abdome a fim de permitir a visualização cirúrgica. Embora seja um gás solúvel em comparação com oxigênio e nitrogênio, pode levar até dois dias para ser absorvido a partir da cavidade peritoneal. Hohlrieder et al. descobriram que a pior dor após a cirurgia laparoscópica ginecológica foi sentida no ombro em $1 \%$ das pacientes, duas horas após a cirurgia, e 70\% das pacientes 24 horas após a cirurgia. Stanley et al. publicaram que a dor atribuída ao gás intraperitoneal era tão frequente quanto a dor da parede abdominal até as primeiras 24 horas, mas diminuiu acentuadamente em 48 horas, juntamente com uma redução correspondente no gás retido. A dor pós-operatória no abdome inferior pode depender da extensão da manipulação intraperitoneal durante a laparoscopia. ${ }^{16}$

A dor torácica por irritação diafragmática pode ser confundida com doença cardíaca coronariana e ser tratada de forma inadequada com anticoagulantes, o que pode produzir um hematoma na ferida ou uma hemorragia intraperitoneal. ${ }^{17}$ Também pode ocorrer enfisema subcutâneo, situação benigna que se resolve sozinha, não sendo preciso nenhum tratamento específico. ${ }^{18}$ No nosso estudo, foram relatados 45 casos de dor, sendo 33 casos de dor leve a moderada e 12 casos de dor intensa.

A infecção da ferida é menos comum após a laparoscopia do que em procedimentos abertos. No entanto, o reconhecimento imediato é importante para evitar aumento da morbidade. $\mathrm{O}$ umbigo é o mais comumente associado à infecção do local cirúrgico do que outros locais de inserção de trocartes, um achado que está correlacionado com o uso do umbigo como um local de extração de espécimes. A deiscência e a hérnia também são importantes complicações pós-operatórias, sendo que esta última deve ser fechada para prevenir obstrução ou estrangulamento do intestino. ${ }^{19}$ No nosso estudo, ocorreram 4 casos de infecção (1 caso de infecção de ferida operatória, 1 caso de infecção urinária, 1 caso de infecção intestinal e 1 caso de sepse abdominal) e ocorreu 1 caso de deiscência.

A incidência de queimaduras foi dramaticamente reduzida pela introdução de coagulação bipolar, porém, ainda existe o perigo de propagação de calor lateral tanto com corrente bipolar como monopolar. É importante garantir que nenhum 
outro órgão esteja em contato ou perto de um órgão ao qual a eletricidade esteja sendo aplicada. A propagação lateral também pode ser minimizada mantendo as lâminas da pinça próximas. Deve sempre ser lembrado que a corrente elétrica é potencialmente perigosa e que todas as regras de segurança para seu uso devem ser rigorosamente obedecidas. ${ }^{17}$ No nosso estudo, nenhuma paciente sofreu algum tipo de queimadura.

O rigor no emprego da técnica cirúrgica no manuseio do instrumental laparoscópico constitui medida simples capaz de prevenir acidentes graves. Mesmo o cirurgião experiente deve estar sempre atento aos detalhes técnicos, além de identificar situações de maior risco (pacientes com cirurgias prévias, obesidade, processos inflamatórios agudos ou recentes, etc.), selecionar adequadamente as pacientes e utilizar manobras e recursos que diminuam a incidência de acidentes que possam ser evitados. ${ }^{9}$

\section{CONCLUSÃO}

Com a evolução das técnicas cirúrgicas, procedimentos invasivos como a laparoscopia emergem como um desafio à Medicina moderna. Os pacientes e os profissionais de saúde estão constantemente buscando melhores resultados, com foco na qualidade de vida no pós-operatório e a longo prazo, sem comprometer a eficiência do tratamento cirúrgico.

A cirurgia minimamente invasiva está ganhando cada vez mais espaço entre os cirurgiões ginecológicos devido aos vários benefícios já citados quando comparados com o mesmo procedimento realizado por via aberta. Fatores limitantes são

\section{REFERÊNCIAS}

1. Yudin MH, Shapiro JL, Weisberg F. Complications of laparoscopic surgery: prevention, diagnosis and management. J Soc Obstet Gynaecol Can. 2000;22(1):10-15.

2. Hurd WW, Falcone T. Gynecologic laparoscopy [Internet]. New York: Medscape; 2015. Acesso em: [12 jan 2017]. Disponível em: https://emedicine.medscape.com/article/265201-overview\#showall

3. Chapron C, Querleu D, Bruhat MA, Madelenat P, Fernandez H, Pierre F, et al. Surgical complications of diagnostic and operative gynaecological laparoscopy: a series of 29.966 cases. Hum Reprod. 1998;13(4):867-72.

4. Gal D, Lind L, Lovecchio JL, Kohn N. Comparative study of laparoscopy vs. laparotomy for adnexal surgery: efficacy, safety, and cyst rupture. J Gyneco Surg. 1995;11(3):153-8.

5. Perugini RA, Callery MP. Complications of laparoscopic surgery. Munich: Zuckschwerdt; 2001 [acesso em: 02 Out 2016]. Disponível em: https://www.ncbi.nlm.nih.gov/books/NBK6923/

6. Barreto A, Bragança JF, Sarian LO, Toledo MC, Sallum LF, Derchain S. Laparoscopia na abordagem inicial de tumores anexiais. Rev Bras Ginecol Obstet. 2014;36(3):124-30.

7. Nascimento R, Eger F. Complicações da cirurgia videolaparoscópica em processos expansivos anexiais benignos. Florianópolis: UFSC; a grande curva de aprendizado da técnica laparoscópica e os custos de material.

Apesar de tudo isso, a laparoscopia consagrou-se com muita rapidez e é um método que será cada vez mais utilizado, devido às suas inúmeras vantagens. A avaliação das cirurgias laparoscópicas que estão sendo realizadas no serviço contribui para a melhoria do atendimento, possibilitando melhor planejamento e treinamento de profissionais.

Todo cirurgião deve estar envolvido na prevenção e no tratamento das complicações da laparoscopia ginecológica, o que é importante para determinar o treinamento dos futuros cirurgiões e para informar às pacientes sobre as possíveis complicações que podem surgir durante e após um procedimento cirúrgico.

A aplicação de técnica cirúrgica apurada durante o manuseio do material laparoscópico e a experiência adquirida ao longo do tempo constituem as principais medidas preventivas para evitar as complicações.

Pode-se concluir que existem complicações inerentes à prática cirúrgica e na cirurgia laparoscópica há complicações específicas desta técnica, estando algumas taxas de complicações do nosso estudo semelhantes e outras menores que as taxas relatadas na literatura mundial. É essencial comunicar nossas complicações para contribuir para um melhor conhecimento sobre elas, assim como classificá-las e registrá-las apropriadamente. A melhor maneira de prevenir complicações cirúrgicas é obter conhecimento de como elas podem ser evitadas.

1999 [acesso em: 19 Jul 2017]. Disponível em: http://www.acm.org. br/revista/pdf/artigos/59.pdf

8. Beleña JM, Nuñez M. Postoperative complications of laparoscopic surgery. Int J Clin Anesthesiol. 2014;2(3):1034.

9. Campos FG, Roll S. Complicações do acesso abdominal e do pneumoperitônio em cirurgia laparoscópica - causas, prevenção e tratamento. Rev Bras Videocirurgia. 2003;1(1):21-8.

10. Berker B, Taskin S, Taskin EA. Complications of laparoscopic gynecologic surgery. [Internet]. Disponível em: http://laparoscopy. blogs.com/prevention_management_3/2010/07/complications-oflaparoscopic-gynecologic-surgery.html.

11. Nogueira C, Ribeiro S, Barata S, Alho C, Osório F, Jorge C. Histerectomia totalmente laparoscópica: análise retrospectiva de 262 Casos. Acta Med Port. 2014;27(1):73:81.

12. Leng J, Lang J, Huang R, Liu Z, Sun D. [Complications in Laparoscopic Gynecologic] Surgery. Chin Med Sci J. 2000, 15(4):222-226.

13. Mbaye M, Cissé ML, Guèye SM, Dièmé ME, Diouf AA, Guèye $\mathrm{M}$, et al. Results of gynecologic laparoscopy in the Central University Hospital (CHU) of Dakar: prospective series of 128 cases. J Am Assoc Gynecol Laparosc. 2001;8(1):68-73. 
14. Sokol AI, Chuang K, Milad MP. Risk factors for conversion to laparotomy during gynecologic laparoscopy. J Am Assoc Gynecol Laparosc. 2003;10(4):469-73.

15. Jackson SA, Laurence AS, Hill JC. Does post-laparoscopy pain relate to residual carbon dioxide? Anaesthesia. 1996;51:485-7.

16. Gibbison B, Kinsella SM. Postoperative analgesia for gynecological laparoscopy. Saudi J Anaesth. 2009;3:70-6.

17. Gordon AG. Complications of laparoscopy. Geneva: Geneva Foundation for Medical Education and Research; 2016 [acesso em: 30 Jun 2017]. Disponível em: https://www.gfmer.ch/Books/ Endoscopy_book/Ch23_Complications_Lap.html

18. Li TC, Saravelos H, Richmond M, Cooke ID. Complications of laparoscopic pelvic surgery: recognition, management and prevention. Human Reproduction. 1997;3(5):505-15.

19. Hindman NM, Kang S, Parikh MS. Common postoperative findings unique to laparoscopic surgery. RadioGraphics. 2014;34(1):119-38.

\section{Como citar:}

Gomes TC, Augusto KL, Machado SL, Amâncio MC, Monteiro GM, Machado LI. Desfechos cirúrgicos e complicações de laparoscopias ginecológicas em hospital universitário brasileiro no período de 2014 a 2016. Rev Med UFC. 2018 out-dez;58(4):33-39. 